\title{
4 Analytical Data and the Life Cycle of Ceramics
}

The ceramics collected in archaeological excavations are usually objects with a long and complex life cycle. As noted by M. Tite (1999), the life cycle begins with the production of the artefact and its subsequent use, maintenance, reuse and amortization processes developed by individuals in the past. Afterwards, once their useful life is complete, the ceramics are discarded and deposited. Pottery vessels can remain in this depositional state throughout long periods of time, until archaeologists dig the sites and recover the objects. During this burial process many agents can potentially produce significant alterations on the original contexts directly related to the behaviours that people carried out in the past.

Closely related to the existence of these diverse processes, authors such as M.Schiffer (1976) distinguished some decades ago between systemic contexts, resulting directly from past human actions, and archaeological contexts. The latter are understood as the records on which archaeologists work and whose formation is influenced by many other processes that take place after the human actions were undertaken in the past. Besides these two contexts and within the life cycle of ceramics, we propose to consider also the actions undertake in the context of heritage management. This context implies, among other aspects, actions for recovering, storing and management of ceramics performed by archaeologists, researchers, restorers and any other professional involved in the chain of actions associated with the processes of excavation, research, conservation and dissemination of the cultural heritage.

Following the proposal referred to above, we must consider that the life cycle of ceramics does not end with the deposition of the pottery in the past but that this cycle of changes continues once the vessels are buried as well as during the management of the materials in present times. Thus, in materialistic terms, pottery is subject to successive physical, chemical and mechanical changes during the several phases involved in their life cycle, from the day the potter selected the clay to the instant the archaeologist recovered the pottery and the curator stored it.

Thereby, the study of the composition of ceramic fabrics and a correct interpretation of the data obtained through the methods discussed requires reflection on the origin of such data. This means to consider the possible alterations that may occur in the various phases of the life cycle of ceramics. These alterations take place during the processes of manufacture, use, deposition, recovering, storage, management and analysis of the ceramics (Buxeda, 1999; De La Fuente, 2008; Tite, 1999, 2008). Therefore, some chemical, mineralogical and textural alterations that may take place in the vessels are addressed in the following paragraphs according to the three main contexts listed above.

a) Systemic Context. Once potters select a raw material with a specific composition, they start to carry out actions along the manufacturing process that alter the original characteristics of the clay. Although, the physicochemical changes that occur in clays 
during the several phases of the production process will be developed in depth in the next chapter, it is advisable to highlight some relevant alterations here. In this sense, when potters add water to the clay in order to make the material plastic and workable they are also altering the chemical composition of the raw material through the introduction of highly soluble salts containing potassium or sodium chloride. Furthermore, the addition of seawater can contribute to increase the amount of chlorine in the ceramics. However, some authors pointed out that the addition of water normally does not distort the relationship between the composition of ceramics and clays (Arnold, 2000; Echallier, 1984).

Potters also usually alter the chemical, mineralogical and textural composition of the raw materials through purification processes such as the manual removal of some non-plastic components. The same effect is obtained by the introduction of external materials to the paste. Thus, the addition of tempers can greatly alter the features of the paste and reduce the compositional differences existing between two vessels produced using distinct raw materials (Blackman, 1992; Kilikoglou et al., 1988; Livingstone-Smith, 2000; Mommsen, 2004; Neff et al., 1988, 1989; Pollard and Heron, 2008; Sterba et al., 2009).

Other apects that distort the relationship between the composition of the ceramics and the raw materials derive from the firing process. At certain temperatures physicochemical reactions occur in the pastes that result in severe changes in their mineralogical and chemical composition. These thermal alterations can greatly alter the composition of the pottery and have to be seriously taken into account to properly interpret the features of the pottery (Arnold, 2000; Buxeda et al., 2002; Kilikoglou et al., 1988; Pollard and Heron, 2008).

Finally, the composition of ceramic artefacts can also be affected by the introduction of new elements in the fabric due to its intended use for cooking, serving and storing liquid and solid products. These kinds of contaminations may increase, for instance, the concentrations of phosphorus (e.g., Bollong et al., 1993; Dunnell and Hunt, 1990; Klein et al., 2004; Maritan and Mazzoli, 2004; Pollard and Heron, 2008) or calcite (Buxeda and Cau, 1995) in the fabric.

b) Archaeological Contexts. Postdepositional alterations in archaeological ceramics are addressed through experimental tests developed under specific conditions and closely controlled variables (e.g., Núñez et al., 2002). In this sense, ceramic bodies are assumed to be initially homogeneous and subsequently, during the burial, certain parts of the vessels such as the surfaces or the margins alter their composition. Therefore, the core is the part of the vessel which usually remains less disturbed by secondary processes (Schwedt et al., 2006). The contamination process is influenced by the more or less saturated composition of the medium in which the ceramic is placed and the porosity index of the pottery. Thus, in a cyclic process of wetting and drying, aqueous solutions filter into the pores of ceramics to subsequently evaporate, generating the crystallization of insoluble salts in the voids. When the crystals that form these salts 
increase in volume they favour the potential formation and propagation of fractures along the ceramic (De La Fuente, 2008; López, 2008; Mommsen, 2004; Pollard and Heron, 2008; Tite, 2008). Besides providing some external elements, these hydrolysis processes promote the neo-formation of mineral phases occurring in the paste, such as calcite (Buxeda and Cau, 1995; Cau et al., 2002) or certain phyllosilicates that usually begin their decomposition during the firing process (Núñez et al., 2002).

After a considerable burial period taphonomical processes that cause important changes in the chemical and mineralogical composition of the ceramics may take place, especially in elements such as $\mathrm{Ca}^{++}, \mathrm{Mg}^{++}, \mathrm{K}^{+}$or $\mathrm{Na}^{+}$which have a greater ability to exchange cations (De La Fuente, 2008; Maritan and Mazzoli, 2004; Pollard et al., 2007; Tschegg, 2009). This means that the composition of the ceramics and the groups statistically established may, in some cases, be also related to postdepositional alterations. In this sense, not all chemical elements have the same range of variation in a group of ceramics. It is often possible to observe wider dispersions in the concentrations of elements such as $\mathrm{CaO}, \mathrm{P}_{2} \mathrm{O}_{5}, \mathrm{~K}_{2} \mathrm{O}, \mathrm{Na}_{2} \mathrm{O}, \mathrm{Ba}, \mathrm{Sr}, \mathrm{Rb}$, As, etc., even if they are measured with high accuracy and low analytical error. In this way, it is common in chemical compositional studies to group the samples without considering many of these elements in the analysis. In most cases, their use is considered to be a risk factor which can greatly distort the chemical groups established and complicate their interpretation (Arnold, 2000; Buxeda et al., 2001, 2002; Cau et al., 2002; Golitko et al., 2012; Ortega et al., 2005; Schwedt et al., 2006; Tite, 2008).

An example of a secondary postdepositional phase occurring in archaeological ceramics is the formation of zeolites (Capel, 1983) such as analcime. This phase, which is detectable by XRD, is associated with calcareous ceramics fired at high temperatures $\left(>1000^{\circ} \mathrm{C}\right)$. Fabrics that have this secondary phase have undergone an alteration process in which results the lixiviation of potassium and rubidium during the firing. Subsequently, during the burial phase, the crystallization of analcime takes place by external contributions of sodium through hydrolysis processes. Eventually, the effect of these processes on the ceramic composition consists in a reduction in the potassium and rubidium concentrations, while the amount of sodium is increased. Recent studies stated that potassium is lost after a long time in the burial phase and not during the firing process, so that the relationship between potassium and rubidium does not seem to be correlated. Anyway, despiteit is quite common to find this kind of alteration in Greek, Punic or Roman pottery (e.g., Buxeda, 1999; Buxeda et al., 2001; Schwedt et al., 2006; Tsantini et al., 2004), analcime is an unusual secondary phase in prehistoric ceramics.

c) Heritage Management Context. Alterations in the composition of ceramics are not restricted to intrinsic factors resulting from the manufacturing and use processes or postdepositional contaminations occurred during burial. There are other extrinsic alterations that result from the processes of management, investigation and preservation of archaeological materials. These contaminations derive, for instance, 
from the recovering, cleaning and storage of ceramics. According to the actions undertaken in these stages, new mineral phases and chemical alterations may generate in pottery vessels. These processes involve, for instance, the contamination of organic residues present in pottery vessels by introducing fatty acids during their handling. Also, the crystallization of salts such as calclacite may take part during the storage of the ceramics (De La Fuente, 2008; Pollard et al., 2007).

Another example would be the fixation of allocthonous chlorine in the pottery due to the use of hydrochloric acid in its cleaning process (Albero, 2013). Such alterations in the materials must be taken into account, especially when pottery vessels stored in museums for long time are selected for analysis. In this way, the criteria used in the management or restoration of the vessels may have changed over time, thus introducing different types of products that alter to different degrees the archaeological ceramics.

Finally, the compositional data may be also influenced by the own analytical process. In this case, we should consider the sampling variance as well as the analytical variance introduced by the detection limits of the instruments. Also, errors due to calibration methods and sample preparation processes should be taken into account. For example, the grain size of the powder used in the XRPD analysis greatly influence the intensity of the peaks obtained in each mineral phase. Also the use of tungsten carbide ball mills alters the concentrations of this element in the samples.

In short, the life cycle of ceramics is long and complex, and we have to realize that it involves several phases and agents that are interrelated. In this regard, the complementary archaeometric methods usually applied to characterize the composition of ceramics provide key information to infer the various changes that occur in the vessels along the different phases of their life. Since we can best approach the physical transformations in the materials and evaluate their causes, we can also undertake more accurate archaeological interpretations of the compositional data. However, the characterization and identification of the possible compositional alterations is not only relevant for undertaking a proper interpretation of the compositional analyses and the technological features of the pottery. Beside this, these analyses provide useful information for all the professionals that work side by side with archaeological heritage. In this way, archaeometric studies are capable of creating synergies and developing good practices between archaeologists, researchers, restorers and curators that work with ceramics in their daily life. 